\title{
EDITORIAL
}

\section{Critical care research in Morocco and Tunisia}

Khalid Abidi ${ }^{{ }^{*}}$ and Lamia Ouanes-Besbes ${ }^{2}$

(C) 2018 Springer-Verlag GmbH Germany, part of Springer Nature and ESICM

\section{Introduction}

Clinical research in the intensive care unit (ICU) has improved our understanding of how various diseases processes impact on patient outcomes [1]. Nevertheless, clinical and health services research in this field is hampered by the heterogeneity of case mix and clinical practice, with relatively small sample sizes [2]. However, there are a number of methods for ICU case mix adjustment and several large observational databases [2]. Large studies are therefore more likely to be published than studies with small samples [3]. However, multicenter research requires a high level of commitment that may be difficult to sustain unless research priorities have been agreed on by participants [2].

Tunisia and Morocco are witnessing a major shift in their epidemiologic profile, with a decline in communicable illness and an increasing burden of non-communicable diseases (e.g., cancer, diabetes, cardiovascular disease, and obesity) which currently account for $75-82.3 \%$ of all deaths [4].

Given the heterogeneous distribution of critical care resources worldwide [5], the deficit is probably greater in developing countries where economies are usually under multiple constraints and a smaller proportion of the gross national income is allocated to healthcare [5]. The World Bank classifies Morocco and Tunisia as lower- to middleincome countries. The total expenditure on health in Tunisia and Morocco is, respectively, US $\$ 785$ and US $\$ 447$ per capita [5]. Priority is given to public health programs and not to expensive services such as critical care [6].

\footnotetext{
*Correspondence: abidikhalid6@gmail.com

${ }^{1}$ Medical ICU, Faculty of Medicine and Pharmacy, Ibn Sina University

Hospital, Mohamed V University, Rabat, Morocco
}

Full author information is available at the end of the article

\section{The current state of research}

A bibliometric analysis was conducted to identify studies published in Morocco and Tunisia. A bibliometric tool (VOSViewer) was used to map the main subjects of research. The most frequently identified critical care medicine research domain in both countries is infectious diseases (Fig. 1). Several retrospective or prospective surveys have been conducted locally to describe the prevalence of infection in ICUs. Most of these studies involved a single center. In addition to these local observational studies, a small number of ICUs have participated in international surveys (Africa, Latin America, Asia, and Europe) of nosocomial infections and antibiotic resistance in the framework of the International Nosocomial Infection Control Consortium [7]. All Tunisian and Moroccan ICUs that have taken part in such surveys are in university hospitals. In the area of respiratory medicine (organ system support and treatment), various departments of critical care medicine have joined European Society of Intensive Care Medicine trial groups (LUNG SAFE Investigators) and the VENTILA Group Consortium in the realization of international observational studies focusing on mechanical ventilation and acute respiratory distress syndrome (incidence, ventilation modalities, and outcome) $[8,9]$.

A second domain of research in which Moroccan and Tunisian ICUs have participated is that into diseases with high local prevalence/specificity. The two topics most frequently covered are severe scorpion envenomation and acute poisoning. Various clinical and experimental studies have provided data on prevalence, benefit from specific serotherapy, acute heart failure, and the use of catecholamine [10]. In addition, various ICUs have focused on diseases related to pregnancy,

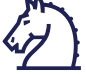 Springer}




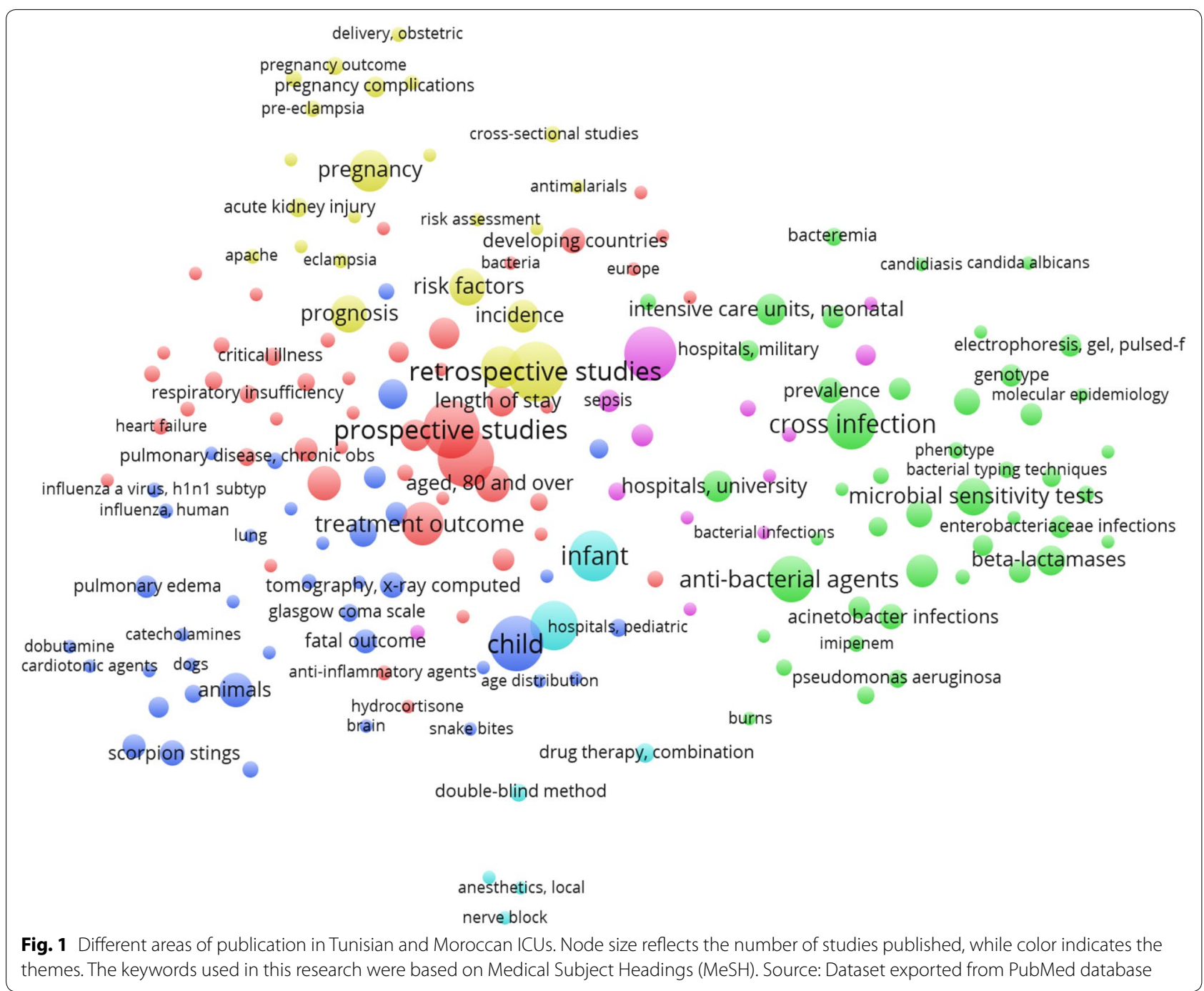

most significantly pregnancy-related acute kidney injury, and their outcome [11]. The domain of respiratory insufficiency has also been evaluated and reported in singlecenter or multicenter surveys. The principal topics have been chronic obstructive pulmonary disease and severe acute asthma.

\section{Barriers to research}

Crucially, there is a lack of local critical care research groups in Tunisian and Moroccan ICUs. The culture of networking is effectively non-existent in medical research in the two countries. Moreover, no institutions or authorities make any effort to concentrate skills in a specific field or around a particular promising research project. There are neither financial incentives nor obligations favoring such cooperation.
In Morocco, there is no real major source of funds for medical research. The research process is complex owing to the partitioning of responsibilities between the Ministry of Health, which is not interested in this process, and the Ministry of Higher Education. As a result, the resources allocated to medical research are very limited in terms of both personnel and funding. A law on the regulation of research was passed in 2015; however, it has not yet been implemented so its impact on research remains unknown. Decisions to limit life support have been studied in Morocco [12]. Religious beliefs and the lack of guidelines and official Moroccan laws could explain ethical limitations on the decision-making process [12].

In Tunisia, there is an older tradition of funding scientific research, spearheaded by health research. In 
2014, Tunisia spent $0.64 \%$ of GDP on scientific research and development (a sharp decrease compared with the years before the Arab Spring), slightly less than Morocco (0.7\%). In clinical research overall, Tunisia ranks 53rd worldwide in terms of absolute number of publications, just ahead of Morocco. For Tunisia, critical care research is among the most active medical disciplines, especially in terms of publication quality.

The structuring of research teams in medicine suffers from being modeled on other scientific fields, with fixed numbers of researchers ( 16 for research units and 24 for laboratories of research). This type of organization is clearly not suited to clinical research as there is no advantage whatsoever to being a researcher in the clinical field. Moreover, funding, originating exclusively from the state via the research ministry, is rudimentary. The funds made available have declined steadily in recent years (zero subsidies for more structures over the past year), while it is almost impossible to fund a research project and/or research network. The legislation on human experimental research dates back to 1991 in line with the country's opening up to international clinical trials sponsored by "big pharma". A recent reform improved the organization, composition and role of ethics and patient protection committees. Once again, it ignored investigator-initiated research, focusing on research with a pharmacological objective. Another ethical aspect of research is end-oflife attitudes. Almost $30 \%$ of deaths occurring in the ICU are preceded by one kind of limitation of life-sustaining support (withholding rather than withdrawing) [13].

\section{Opportunities and needs}

Priority needs to be given to advancing research in Tunisian and Moroccan ICUs. Various strategies can be adopted. First, in the light of our findings, we should encourage the development of a culture of networking in our ICUs. This goal should be realized by creating various national and North-African collaborative groups and conducting investigator-initiated multicenter critical research under the auspices of local societies of critical care in both countries. The networking can be used as a platform for further collaborative studies. The mission of these groups will be the structuring of medical research, the fulfillment of common research objectives, the development of protocols, and improvement of the outcomes of critical illness.

Second, funds must be found to enable the conduct of research. The structure of ICUs in Morocco and Tunisia need not be a hindrance to performing and interpreting medical research. The national governments, via the respective Ministry of Health and/or Ministry of Higher Education, should provide sufficient funds and human resources (residents and nurses) and motivate study investigators with grants or remuneration. Experienced investigators can carry out high-quality multicenter studies even with small budgets. The success and the productivity of a number of collaborative groups with limited resources demonstrate how academic motivation is not determined only by research funding and how good ideas can be more important than money [1]. Ideally, the human and financial cost of critical illness, the funding required for the conduct of studies in the ICU, and the potential benefits to society would be considered by the policymakers responsible for allocating funds [1]. Independently of the involvement of government in this project funding, national ICU societies should establish partnerships with pharmaceutical and medical technology companies. In addition, the national societies/governments should prioritize pragmatic and high-impact clinical research, use funding for research according to advance planning, and establish setting-specific research priorities with focus on high-impact, low-cost initiatives [5]. Third, reliable and efficient critical care data repositories should be created for the advancement of medical research [14].

\section{Author details \\ ${ }^{1}$ Medical ICU, Faculty of Medicine and Pharmacy, Ibn Sina University Hospital, Mohamed V University, Rabat, Morocco. ${ }^{2}$ Intensive Care Unit, CHU Fetouma \\ Bourguiba, University of Monastir, Monastir, Tunisia.}

Compliance with ethical standards

Conflicts of interest

The authors report no conflict of interest.

Received: 17 January 2018 Accepted: 14 February 2018 Published online: 8 March 2018

\section{References}

1. Cook D, Brower R, Cooper J, Brochard L, Vinecnt JL (2002) Multicenter clinical research in adult critical care. Crit Care Med 30:1636-1643

2. Goldfrad C, Vella K, Bion JB, Rowan KM, Black NA (2000) Research priorities in critical care medicine in the UK. Inntensive Care Med 26:1480-1488

3. Easterbrook PJ, Berlin JA, Gopalan R et al (1991) Publications bias in clinical research. Lancet 337:867-872

4. World Health Organization. Global Health Observatory. Available at http://apps.who.int/nha/database/key_indicators/index/en. Accessed May 2016

5. Society of Critical Care Medicine: A Closer look at Critical Care Worldwide. 2014. Available at: http://www.sccm.org/communications/Critical-connections/Archives/Pages/A-Closer-Look-at-Critical-Care-Worldwide.aspx. Accessed May 8, 2016

6. Arabi YM, Schultz MJ, Salluh JIF (2017) Intensive Care Medicine in 2050: global perspectives. Intensive Care Med 43(1695-1695):00

7 Rosenthal VD, Maki DG, Mehta Y, Leblebicioglu H, Memish ZA et al (2014) International Nosocomial Infection Control Consortium (INICC) report, data summary of 43 countries for 2007-2012. Device-associated. Module. Am J Infect Control 42:942-956

8 Nin N, Muriel A, Peñuelas O, Brochard L, Lorente JA et al (2017) Severe hypercapnia and outcome of mechanically ventilated patients with 
moderate or severe acute respiratory distress syndrome. Intensive Care Med 43:200-208

9 Laffey JG, Bellani G, Pham T, Fan E et al (2016) Potentially modifiable factors contributing to outcome from acute respiratory distress syndrome: the LUNG SAFE study. Intensive Care Med 42:1865-1876

10 Elatrous S, Ouanes-Besbes L, Ben Sik-Ali H, Hamouda Z, BenAbdallah S, Tilouche N, Jalloul F, Fkih-Hassen M, Dachraoui F, Ouanes I, Abroug F (2015) Study of severe scorpion envenoming following subcutaneous venom injection into dogs: hemodynamic and concentration/effect analysis. Toxicon 104:1-6

11 Bentata Y, Madani H, Berkhli H, Haddiya I, Saadi H, Mimouni A, Housni B (2015) Acute kidney injury according to KDIGO stages and maternal mortality in the intensive care unit. Intensive Care Med 41:555-556
12 Damghi N, Belayachi J, Aggoug B et al (2011) Withholding and withdrawing life-sustaining therapy in a Moroccan emergency department: an observational study. BMC Emerg Med 11:12

13 Ouanes I, Stambouli N, Dachraoui F et al (2012) Pattern of end-of-life decisions in two Tunisian intensive care units: the role of culture and intensivists' training. Intensive Care Med 38:710-717

14 O'Connor S, Ayres A, Cortellini L, Rosand J, Rosenthal E, Kimberly WT (2012) Process improvement methods increase the efficiency, accuracy, and utility of a neurocritical care research repository. Neurocrit Care 17:90-96 